\title{
The Effect of Erythropoietin Treatment on Gene Expression Profile of Mesenchymal Stem Cells
}

\author{
Dilara Dalkiran"1, [MSc] \\ ORCID: 0000-0003-0992-7892 \\ Beren Karaosmanoglu' ${ }^{1,2,}[\mathrm{PhD}]$ \\ ORCID: 0000-0001-5564-4813 \\ Assist. Prof. Ekim Z. Taskiran ${ }^{2,}$, [PhD] \\ ORCID: 0000-0001-6040-6625
}

1, Department of Stem Cell Sciences, Institute of Health

Sciences, Hacettepe University.

2, Department of Medical Genetics, Faculty of

Medicine, Hacettepe University.

* Corresponding Author: Ekim Z. Taskiran

Address: Hacettepe University Faculty of Medicine

Department of Medical Genetics, Sihhiye, Ankara,

Turkey.

Phone: +90 3123052636

Fax: +90 3123115522

E-mail: eztaskiran@hacettepe.edu.tr,

ekimtaskiran@gmail.com

\section{we ABSTRACT Com}

Introduction: Mesenchymal Stem Cells are one of the most important elements of bone marrow microenvironment, which has a role in stromal support and stem cell differentiation. Exosomes are small vesicles that responsible from various cellular roles such as cell-cell communication and cell signaling, which may affect nearby and distant cells/tissues. Mesenchymal Stem Cells have therapeutic importance because of their multipotency and immune modulation potentials also with their exosomes. Erythropoietin, produced by liver hepatocytes, is responsible for erythroid differentiation (erythropoiesis) in bone marrow. In addition, Erythropoietin treatment of several cell types including Mesenchymal Stem Cells, showed therapeutic effects in various diseases.

Objective: The aim of this study is to examine the effect of erythropoietin on bone marrow Mesenchymal Stem Cells transcriptome and exosome derived miRNA profile.

Materials and Methods: Effect of 3 different doses of Erythropoietin (1 IU/ml, 10 IU/ $\mathrm{ml}$ and $100 \mathrm{IU} / \mathrm{ml}$ ) for 48 hours on Mesenchymal Stem Cells transcriptome profile was analyzed. The results illustrated that $10 \mathrm{IU} / \mathrm{ml}$ Erythropoietin treatment has the most effective concentration in terms of gene expression profile. Therefore, small RNA libraries targeting

miRNA was analyzed with $10 \mathrm{IU} / \mathrm{ml}$ Erythropoietin treated versus non treated groups with next generation sequencing.

Results: We found that Erythropoietin treatment slightly changed global gene expression profile. On the other hand, it was observed that Erythropoietin treated Mesenchymal Stem Cells have different exosomal miRNA profile.

Conclusion: Differentially expressed exosomal miRNAs may have therapeutic effects in different conditions. It will be important to perform further studies with in vitro models, mimicking different physiological conditions and diseases for Mesenchymal Stem Cells and exosome biology.

Key words: Mesenchymal stem cells (MSCs), erythropoietin (Epo), transcriptomics, exosome, miRNA.

Received: 9 May 2019, Accepted: 20 May 2019, Published online: 30 June 2019

\section{INTRODUCTION}

Mesenchymal Stem Cells (MSCs), which have multipotent character, have the capacity to differentiate into mesoderm-derived bone, fat, cartilage and muscle cells [1]. MSCs were positive for surface markers CD90, CD73, and CD105 and negative for CD34, CD45, and CD14 [1]. MSCs are one of the most main members of the bone marrow microenvironment. 16
Not only does it support stromal support, but it also affects stem cell fate, such as differentiation, proliferation and staying quiescent with secreted cytokines [2]. Not only the MSCs themselves but also the molecules they secrete provide a therapeutic effect [3]. One of the most important biological tools that have this effect are vesicles which called 
extracellular vesicles [3]. These vesicles include exosomes and microvesicles. Exosomes are small vesicles of 50-100 nm size [4]. They are involved in cellcell communication and cell signaling. This mechanism affects the metabolism of nearby and distant cells or tissues [3]. The exosomes are also rich and varied in terms of content and can carry many types of molecules such as growth factors, signal lipids, mRNAs and miRNAs [5]. In previous studies, MSCderived exosomes have been analyzed in many different diseases and tissues, and it has been observed that it supports the proliferation and migration of tissue-specific stem cells that accelerates the healing of scar tissues, protects various tissues and decreases the inflammatory effect, protects against apoptosis and crosses the blood brain barrier [6-8].

Transplantation of exosomes, which are the most important elements of MSCs to produce therapeutic effects, has the potential to be a safe option. It is thought that there is a direct relationship between the effect and content of exosomes. They provide a therapeutic effect with mRNA and miRNAs involved in gene expression control. [9]. In this way, it is thought that the therapeutic effect of MSCs can be mimicked by exosomes, avoiding the risks of transplanting MSCs [10].

Human erythropoietin (Epo) hormone is a glycosylated protein weighing $30.4 \mathrm{kDa}$ [11]. Epo is produced by liver hepatocytes during embryonic development. It is produced by peritubular capillary endothelial cells in the liver and kidney in the adult period after birth [12]. However, peritubular fibroblast-like cells in the cortex of the kidney are responsible for the production of mostly large parts of the kidney [12]. Epo gene encoding erythropoietin is found in chromosome 7q22 region [13], and has an oxygen-dependent expression [12]. Epo, a glycoprotein hormone, allows the differentiation of hematopoietic stem cells in the bone marrow into erythroid precursor cells (erythropoiesis) [14]. This function is stimulated through the receptor called EpoR.

In previous studies, it was observed that different cell types such as neuron, endothelium, and cardiomyocytes were started to express EpoR as a result of treatment with Epo [12]. This suggests that Epo is also effective in addition to erythropoiesis. Although the role of Epo in erythropoiesis is the most well-known, it has been observed in the studies conducted that it also has an effect on hematopoietic cells, macrophages and endothelial cells [15]. It has also been shown in studies that Epo has an effect on neural, muscle and cardiovascular tissues.
For example, by the effects of increasing the expression of antioxidant enzymes and reducing the release of free radicals, the brain also shows neutrophic and neuron protective properties [16].

Various Epo doses have been tried in different disease models and various effects have been observed in many studies [17-19]. The study by Wang et al. in 2015, it was thought that the Epo treatment on MSCs that help protect kidney function from chronic kidney diseases will increase their effect [17]. This positive result was thought to be related to the load of microvesicles released from MSCs. The effect of Epo on these microvesicles and their protective function in chronic kidney diseases were investigated [17]. Microvesicles obtained from MSCs that were treated with Epo at different concentrations (1, 10,100 , and $500 \mathrm{IU} / \mathrm{ml}$ ), and microvesicles obtained from MSCs that are not treated with Epo were used in the treatment of kidney damage, both in vivo and in vitro [17]. In the dose range of $1-100 \mathrm{IU} / \mathrm{ml}$ Epo, a dose-dependent increase in the microvesicles was observed. Microvesicles obtained from MSCs treated with Epo have been shown to have a superior protective effect, particularly in fibrosis-associated kidney injury [17]. In a microarray study to examine the miRNA profile, it was observed that the expression of 212 miRNA was increased. It has been shown that the changes have a protective effect on renal function.

Treatment of mesenchymal stem cells with erythropoietin has been studied by many researchers and has been shown to increase the therapeutic effect. However, these studies were limited because they examined the effect on specific genes. In this study, the alteration of the gene expression profile of mesenchymal stem cells caused by erythropoietin will be examined with a more detailed and broad perspective by revealing the transcriptome profile, and exosomal miRNAs.

\section{MATERIALS and METHODS}

\section{Cell Culture and Erythropoietin (Epo) Treatment}

Human bone marrow derived mesenchymal stem cells (BM-MSCs) were purchased from American Type Culture Collection (ATCC, Cat. No: PCS-500012TM, Lot No: 63208778), which were obtained from 24 years old Caucasian male bone marrow donor. BM-MSCs were cultured in DMEM-LG (supplemented with $10 \%$ FBS, $1 \%$ penicillin-streptomycin and $1 \% \mathrm{l}$-glutamine) and $37^{\circ} \mathrm{C}, 5 \% \mathrm{CO}_{2}$. Culture medium was changed twice a week; passage 3 cells 
were used for further experiments.

MSCs were seeded on a 6-well cell culture plates with density of 40.000 cells per $\mathrm{cm}^{2}$. After reaching 70$80 \%$ confluency 1,10 and $100 \mathrm{IU} / \mathrm{ml}$ Erythropoietin (Epo) (Prospec Cyt-325) was added into culture medium. All samples were seeded in triplicates and incubated for 48 hours at $37^{\circ} \mathrm{C}, 5 \% \mathrm{CO}_{2}$. Culture medium was collected for exosome isolation. For exosome studies, culture medium was prepared with exosome-depleted FBS (Thermo Fisher Scientific).

\section{Total RNA, Exosome and Exosomal miRNA Isolation}

Culture medium was removed (collected for exosome isolation), cells were washed with PBS, $1 \mathrm{ml}$ TRIzol (Sigma) was added and RNA was isolated according to the manufacturer's instructions. RNA quality and quantity were measured with spectrophotometer (Nanodrop 2000, Thermo Fisher Scientific).

Culture medium with $10 \mathrm{IU} / \mathrm{ml}$ Epo was collected and exosome isolation was performed with Total Exosome Isolation Solution (Cat. No: 4478359, Thermo Fisher Scientific) according to the manufacturer's instructions. Exosome pellet was resuspended in PBS and kept at $-20^{\circ} \mathrm{C}$ for further experiments. miRNA was isolated with Total Exosome RNA and Protein Kit (Cat. No: 4478545, Thermo Fisher Scientific) according to the manufacturer's instructions with small RNA enrichment protocol. miRNA pellet was resuspended in $15 \mu \mathrm{I}$ TE buffer. These experiments were carried out in three biological replicates and the samples were continued by combining after the exosome isolation.

\section{Quantitative Transcriptomics and Exosomal miRnA Profiling}

cDNA was synthesized from $10 \mu \mathrm{l}$ of each RNA samples with VILO Superscript CDNA synthesis Kit (Thermo Fisher Scientific). The libraries were prepared by using the Ampliseq Transcriptome Human Gene Expression kit (Thermo Fisher Scientific) and the Ion Chef instrument (Thermo Fisher Scientific). All samples were studied in duplicates. On the other hand, for exosomal miRNA profiling, lon Total RNASeq Kit (version 2) was used for library preparation. After library preparation, clonal amplification was performed with lon PI Hi-Q OT2 200 kit (Thermo Fisher Scientific). The next generation sequencing reaction was performed on the Ion Proton device (Thermo Fisher Scientific) by using the Ion PI Hi-Q Sequencing 200 Kit (Thermo Fisher Scientific). For data analysis, all subsequent reads were normalized by the "read per millions" method and experimental groups were analyzed comparatively. Reads were mapped to the human genome assembly hg19. Differentially expressed genes (DEG) were determined with iDEP, fold changes $\log _{2} 2$ (4-fold) and above were considered as significant and FDR cutoff was 0.1 .

\section{RESULTS}

\section{Epo Treatment Affect the Gene Expression Profile of MSCs}

MSCs were treated with 3 different concentrations $(1 \mathrm{IU} / \mathrm{ml}, 10 \mathrm{IU} / \mathrm{ml}$ and $100 \mathrm{IU} / \mathrm{ml}$ ) of Epo. Epo-treated and untreated MSCs were comparatively analyzed, $10 \mathrm{IU} / \mathrm{ml}$ Epo treated has the most DEG numbers (Figure 1). Gene expression changes (at least 4-fold and above) which are dependent on $10 \mathrm{lU} / \mathrm{ml}$ Epo treatment are listed in Tables 1 and 2. In this analysis, it was shown that the expression of 82 genes increased and the expression of 86 genes decreased as a result of $10 \mathrm{IU} / \mathrm{ml}$ Epo treatment. Therefore, this concentration $(10 \mathrm{lU} / \mathrm{ml})$ was selected for further experiments.

Figure 1: Analysis results of Epo-treated and untreated MSCs.Differentially expressed gene numbers (DEGs) of Epo treated groups with respect to control MSCs.

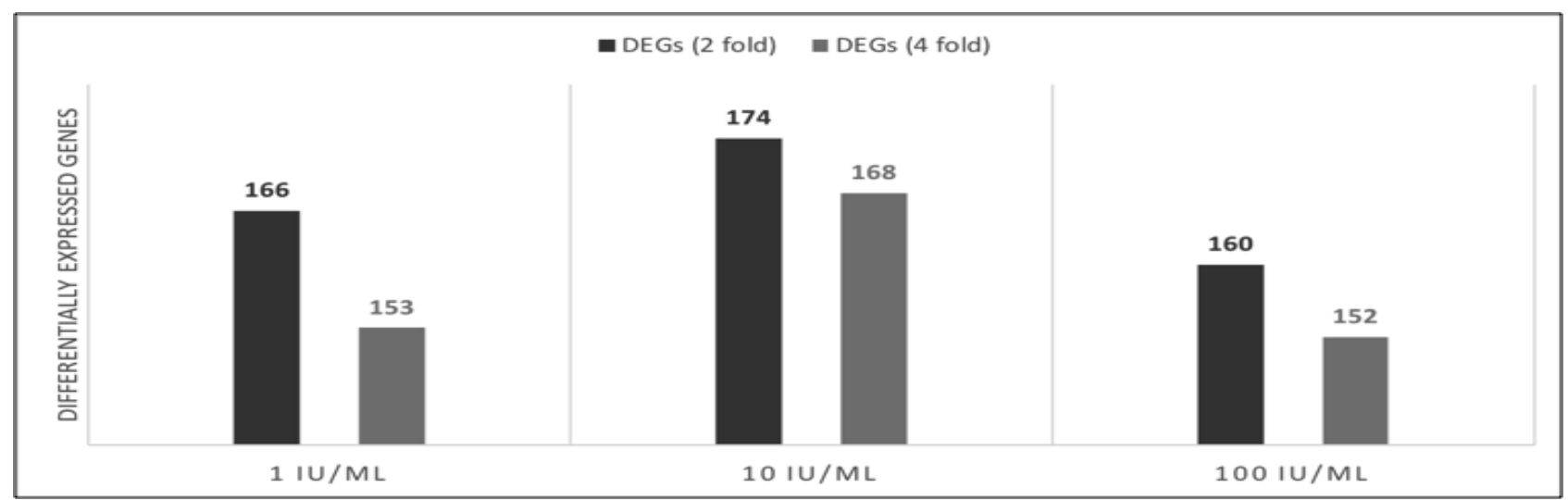


Table 1: $\log _{2} 2$ (4-fold) increased gene list after $10 \mathrm{IU} / \mathrm{ml}$ Epo treatment. Genes were listed alphabetically.

\begin{tabular}{|l|c|c|c|c|}
\hline AA06 & FAM159B & IL3 & NFATC4 & SH2D3C \\
ACTL9 & FAM180B & IL31RA & NOXRED1 & SLC15A2 \\
ADRA2B & FAM222A & IQSEC3 & NPC1L1 & SLC18A3 \\
BHLHA15 & FAM71A & KIAA1804 & NR2E3 & SLC6A4 \\
BPI & FBP1 & KIF12 & OR10V1 & SNX10 \\
C12ORF70 & FGF11 & KRT37 & PANX2 & STAR \\
C12ORF77 & FGF12 & KYNU & PDZD2 & TMED6 \\
C3ORF45 & FGFBP3 & LCN2 & PDZD7 & TMLHE-AS1 \\
C8G & FLJ34503 & LEKR1 & PKLR & TRIML2 \\
CPA6 & FRAS1 & LINC00111 & PLA2G2A & UTS2R \\
DCSTAMP & GFI1 & LINC00176 & PRLR & VANGL2 \\
DLEC1 & GOLGA7B & LINC00547 & PSG1 & WDR86 \\
DLG3 & HAPLN4 & LRRC61 & RAB40A & WEE2 \\
DLL3 & HMP19 & LUZP2 & RASAL3 & XIRP1 \\
DLL4 & HRASLS & NACA2 & RFPL3 & ZBP1 \\
EEF1A2 & IGF1 & NEK5 & RINL & ZMAT1 \\
ERVMER34-1 & IL27 & & & \\
\hline
\end{tabular}

Table 2: $\log _{2} 2$ (4-fold) decreased gene list after $10 \mathrm{IU} / \mathrm{ml}$ Epo treatment. Genes were listed alphabetically.

\begin{tabular}{|l|c|c|c|c|}
\hline ALPK2 & CDH3 & KEL & NR0B1 & SLAMF8 \\
ANGPT2 & CDK18 & KRTAP5-11 & NR6A1 & SLC13A5 \\
ANO2 & CHST13 & LILRA5 & OR5AP2 & SPRYD4 \\
ANXA8 & CKMT1A & LOC100133669 & PARD6A & ST6GALNAC5 \\
APOBEC3A & COLEC10 & LOC100499183 & PCDH9 & SVOPL \\
ARHGAP15 & CYP21A2 & LOC100507034 & PI3 & SYT2 \\
ARID3C & DCAF8L2 & LOC255130 & PIP5K1B & TBXAS1 \\
ATAD3C & DOK6 & LOC283050 & PRDX1 & TFF2 \\
ATRNL1 & DRD4 & LOC387895 & PRM3 & TMEFF2 \\
BEST1 & DTX4 & LOC402160 & PXDNL & TMEM163 \\
BIN2 & FAM162B & LRG1 & RAB24 & TMEM244 \\
C21ORF62 & GIP & MATK & RAD51B & TMPRSS9 \\
CA5A & GUCY1A2 & MRGPRE & RHCE & TRIM7 \\
CACNG7 & HEATR4 & MTMR8 & SAA2 & UGT8 \\
CADPS & HLA-J & MYF6 & SEMA4D & UPK1B \\
CARNS1 & IL20RA & NOD2 & SERPINB3 & VNN2 \\
CCL4 & ILDR2 & NOSTRIN & SFMBT2 & ZNF219 \\
CDC42BPG & & & & \\
\hline
\end{tabular}




\section{Altered Exosomal miRNA Profile of MSCs After Epo Treatment}

We hypothesized that the Erythropoietin might influence the exosomal contents of MSCs. We analyzed the exosomal miRNAs in MSCs before and after Epo treatment. We found critical changes in miRNA profile. As shown in Table 3 , some of the miRNAs were only found in Epo $(10 \mathrm{lU} / \mathrm{ml})$ treated exosomes, but not in untreated group. It was noted that although 4 miRNAs were present in the control group, they were not in exosomes as a result of Epo treatment (Table 4). However, after Epo treatment, there was a decrease in 14 miRNA and an increase in 2 miRNAs compared to exosomes isolated from untreated group (Table 5).

Table 3: Exosome related miRNAs after $10 \mathrm{IU} / \mathrm{ml}$ Epo treatment.

\begin{tabular}{|c|c|c|c|}
\hline miRNA & miRNA Sequence & Read Count (Control) & $\begin{array}{l}\text { Read Count } \\
\text { (10 IU/ml Epo) }\end{array}$ \\
\hline hsa-miR-16-5p & UAGCAGCACGUAAAUAUUGGCG & 0 & 151 \\
\hline hsa-miR-140-5p & CAGUGGUUUUACCCUAUGGUAG & 0 & 123 \\
\hline hsa-miR-99a-5p & AACCCGUAGAUCCGAUCUUGUG & 0 & 107 \\
\hline hsa-miR-199a-3p & ACAGUAGUCUGCACAUUGGUUA & 0 & 86 \\
\hline hsa-miR-199b-3p & ACAGUAGUCUGCACAUUGGUUA & 0 & 86 \\
\hline hsa-miR-27b-3p & UUCACAGUGGCUAAGUUCUGC & 0 & 64 \\
\hline hsa-miR-455-5p & UAUGUGCCUUUGGACUACAUCG & 0 & 46 \\
\hline hsa-miR-376c-3p & AACAUAGAGGAAAUUCCACGU & 0 & 45 \\
\hline hsa-miR-423-3p & AGCUCGGUCUGAGGCCCCUCAGU & 0 & 44 \\
\hline hsa-miR-574-3p & CACGCUCAUGCACACACCCACA & 0 & 43 \\
\hline hsa-miR-93-5p & CAAAGUGCUGUUCGUGCAGGUAG & 0 & 37 \\
\hline hsa-miR-485-3p & GUCAUACACGGCUCUCCUCUCU & 0 & 33 \\
\hline hsa-miR-574-5p & UGAGUGUGUGUGUGUGAGUGUGU & 0 & 30 \\
\hline hsa-let-7a-5p & UGAGGUAGUAGGUUGUAUAGUU & 0 & 28 \\
\hline hsa-let-7g-5p & UGAGGUAGUAGUUUGUACAGUU & 0 & 24 \\
\hline hsa-let-7c-5p & UGAGGUAGUAGGUUGUAUGGUU & 0 & 23 \\
\hline hsa-miR-199a-5p & CCCAGUGUUCAGACUACCUGUUC & 0 & 22 \\
\hline hsa-let-7f-5p & UGAGGUAGUAGAUUGUAUAGUU & 0 & 15 \\
\hline hsa-miR-126-3p & UCGUACCGUGAGUAAUAAUGCG & 0 & 14 \\
\hline
\end{tabular}

Table 4: Exosome related miRNAs after $10 \mathrm{lU} / \mathrm{ml}$ Epo treatment.

\begin{tabular}{|l|c|c|c|}
\hline miRNA & miRNA Sequence & Read Count (Control) & Read Count (10 IU/ml Epo) \\
hsa-miR-19a-3p & UGUGCAAAUCUAUGCAAAACUGA & 321 & 0 \\
hsa-miR-103a-3p & AGCAGCAUUGUACAGGGCUAUGA & 273 & 0 \\
hsa-let-7i-5p & UGAGGUAGUAGUUUGUGCUGUU & 241 & 0 \\
hsa-miR-151a-5p & UCGAGGAGCUCACAGUCUAGU & 114 & 0 \\
\hline
\end{tabular}


Table 5: Altered miRNAs depending on $10 \mathrm{IU} / \mathrm{ml}$ Epo treatment.

\begin{tabular}{|c|c|c|c|}
\hline miRNA & miRNA Sequence & Read Count (Control) & $\begin{array}{l}\text { Read Count } \\
\text { (10 IU/ml Epo) }\end{array}$ \\
\hline hsa-miR-145-5p & GUCCAGUUUUCCCAGGAAUCCCU & 2232 & 51 \\
\hline hsa-miR-24-3p & UGGCUCAGUUCAGCAGGAACAG & 2066 & 230 \\
\hline hsa-miR-23a-3p & AUCACAUUGCCAGGGAUUUCC & 2008 & 119 \\
\hline hsa-miR-195-5p & UAGCAGCACAGAAAUAUUGGC & 1335 & 101 \\
\hline hsa-miR-26a-5p & UUCAAGUAAUCCAGGAUAGGCU & 1033 & 43 \\
\hline hsa-miR-10b-5p & UACCCUGUAGAACCGAAUUUGUG & 930 & 7 \\
\hline hsa-miR-214-3p & ACAGCAGGCACAGACAGGCAGU & 804 & 62 \\
\hline hsa-miR-140-3p & UACCACAGGGUAGAACCACGG & 795 & 266 \\
\hline hsa-let-7b-5p & UGAGGUAGUAGGUUGUGUGGUU & 473 & 118 \\
\hline hsa-miR-125b-5p & UCCCUGAGACCCUAACUUGUGA & 322 & 17 \\
\hline hsa-miR-320a-3p & AAAAGCUGGGUUGAGAGGGCGA & 321 & 77 \\
\hline hsa-miR-143-3p & UGAGAUGAAGCACUGUAGCUC & 278 & 174 \\
\hline hsa-miR-29a-3p & UAGCACCAUCUGAAAUCGGUUA & 262 & 1 \\
\hline hsa-miR-451a & AAACCGUUACCAUUACUGAGUU & 256 & 3 \\
\hline hsa-miR-21-5p & UAGCUUAUCAGACUGAUGUUGA & 17 & 59 \\
\hline hsa-miR-101-3p & UACAGUACUGUGAUAACUGAA & 2 & 61 \\
\hline
\end{tabular}

\section{DISCUSSION}

In this study, the effects of erythropoietin on MSCs from bone marrow were investigated at the level of gene expression. Transcriptome and exosome-derived miRNA profiles of Epo treated MSCs were investigated using the next generation sequencing method. It is important to clarify the content of these exosomes, as it is shown in previous studies that MSC-induced exosomes may have a therapeutic potential [3-5].

According to the previous studies, 3 different Epo doses were selected $[17,20]$. In the first part of the study, the effect of 48 hours of Epo treatment on 3 different concentrations $(1 \mathrm{IU} / \mathrm{ml}, 10 \mathrm{IU} / \mathrm{ml}$ and 100 $\mathrm{IU} / \mathrm{ml}$ ) of Epo transcriptome profile was investigated. Expression changes were generally found to be of low copy transcripts. In other words, selected Epo concentrations result in minimal transcriptomic differences in MSCs at 48 hours of incubation. According to comparative gene expression analysis, $10 \mathrm{IU} / \mathrm{ml}$ Epo treatment has found to be the highest number of DEGs. Therefore, in the second part of the study, this concentration $(10 \mathrm{lU} / \mathrm{ml})$ was used to investigate the effect of Epo treatment on miRNA expression originated from exosomes. In order to investigate the effect of Epo treatment on the miRNA profile in exosomes of MSCs, exosome isolation and subsequent RNA isolation from MSCs cultured with Epo at a concentration of $10 \mathrm{IU} / \mathrm{ml}$ for 48 hours were performed. By using next generation sequencing method, small RNA libraries targeting "miRNAs" were investigated under Epo treatment. The comparative analysis revealed a change in the number of several different miRNAs. Nineteen miRNAs were found to be present in the exosomes due to Epo treatment, but not in the control samples (Table 3), and it was noted that although 4 miRNAs were present in the control group, they were not in exosomes as a result of Epo treatment (Table 4). However, the reading number of 14 miRNAs decreased due to Epo, while 2 miRNAs increased (Table 5).

As a result of these analyzes, it is noteworthy that changes, especially in more than one element of the Let-7 miRNA family, were observed (Table 3). This miRNA family has different tasks and targets in various tissues and cells. Let-7e-5p and Let-7f-5p found in MSC originated from exosomes as a result of Epo treatment are known to support angiogenesis $[12,15]$. Let-7g-5p that are detected in the MSCexosomes due to the Epo effect has been shown to be negatively regulating events such as angiogenesis, inflammation, migration, and senescence [21]. Let-7i-5p, which is not found in exosomes originated 
in MSC as a result of Epo treatment positively affects its expression by bonding to TATA motive in the promotor region of IL-2, which triggers inflammation in CD4 positive T cells [22]. However, it may also act to trigger the immune system by a change in the microenvironment caused by disease and/or the cell type it is in. In another study, Let-7i-5p was reported to suppress apoptosis via FASL inhibition [23]. Let$7 b-5 p$, which was found to decrease as a post-Epo cargo, has been shown to positively affect angiogenesis in the heart by targeting TGFBR1 in the endothelial cells [24]. When Let-7 miRNA family members with an increase/decrease in the exosomes content are examined, it can be said that a result of Epo treatment results in a miRNA profile in favor of angiogenesis.

Some of the exosomes of miRNAs are associated with tumor biology. It has been shown that miR-140$5 p$, which begins to be transported on exosomes due to Epo treatment, plays a role as a tumor suppressor [25]. Another miRNA with similar effects and gets triggered with Epo treatment is miR16-5p. It supports apoptosis by targeting $\mathrm{Bcl} 2$. Another miRNA cargo associated with cancer is miR-99a- $5 \beta$ detected in exosomes due to Epo treatment. This molecule has been shown to increase miR-99a-5p expression of leukemia stem cells to resist chemotherapy in acute myeloid leukemia (AML) and has the potential to become a biomarker for AML [26]. miR485-3p, found in exosomes as a result of Epo treatment, controls the expression of DNA topoisomerase 2 molecule, which is targeted by many chemotherapy drugs, in the negative direction by targeting Nuclear transcription factor $Y$ subunit beta (NF-YB). Decreased miR-485-3p expression in lymphoblastic leukemia is a mechanism used to provide drug resistance [27].

Anti-tumor effects of the molecules, in whose miRNA profiles a reduction of the exosomes was observed (miR-10b-5p, miR-145-5p, miR-140, miR195$5 p$, miR29a-3p, mir-26a-5p, and miR-451), has been reported. It has been shown that these molecules have negative effects on cell proliferation, invasion, and migration while having apoptosis-promoting effects. [28-34]. Contribution to tumor development may be shown as a common feature of miRNAs, which are not included in the exosomes as a result of Epo treatment (miR151a-5p, miR-103a-3p, miR-19a-3p). These miRNAs act in favor of tumor development by suppressing molecules that negatively affect tumor growth. For this reason, in many types of cancers, increased expressions are found [35-37].

Some of the miRNAs detected in the study are associated with the immune system. It is noteworthy that miR-19-3p, which is not involved in exosomes as a result of Epo treatment, is involved in communication between the natural and acquired immune system. It exhibits immunosuppressive properties by targeting the 5-Lipoxygenase (5-LO) enzyme produced by myeloid cells, responsible for the synthesis of leukotriene. But if miR-19a-3p and miR-125b-5p expressions are inhibited, it indicates the potential for initiation of inflammation if the myeloid cells are present in the target area [38]. mir-19$3 p$ works to reduce tumor development and invasion in breast cancer. By reducing the expression of Fos-related antigen-1 (Fra-1), negatively affects the anti-inflammatory M2 macrophage phenotype and polarization. In this way, it activates the immune system to fight against breast cancer [37].

Among the detected miRNA molecules, those associated with stem cell biology and regeneration were also noted. miR-26a-5p has been shown to target iNOS and suppress expression in human cartilage cells [30]. In terms of this mechanism, the effect of Epo on pathological conditions such as osteoarthritis is worth examining. miR-199a-3p is a molecule that is detected in exosomes in the presence of Epo and stands out due to its function. This miRNA, whose expression is controlled by p53, allows the heart muscle cells that exited the cell cycle in the adult period, to re-enter the cell cycle [39]. It not only does help the heart muscle cells avoid apoptosis, but also supports their proliferation [40]. In this way, it has great potential to repair the damage in the heart, which cannot be regenerated, caused by heart attack or other reasons.

What makes this study original is the fact that miRNA content of exosomes collected by treatment of human bone marrow-derived MSCs with Epo is analyzed using the next generation sequencing technology. In addition, the exosome-specific miRNA analysis and the usage of next generation sequencing resulted in highly reliable and sensitive results. Although minimal amount of changes was detected at the transcriptomic level in the earlier studies, the fact that the expression change in miRNA in the exosome cargo was determined is critical in terms of the targets of the miRNA originated in exosomes. The fact that miRNAs with changes in exosomal cargo were found to play a role especially 
in critical biological diseases and pathways, such as cancer, angiogenesis, cell migration/invasion, are critical for the widespread influence of the data obtained in this study. The therapeutic potential of the detected miRNAs will shed light on the interdisciplinary studies that may occur in the future.

The potentials of the miRNAs identified in this study may be used as biomarkers in various experimental/ pathological conditions should also be investigated and their specific functions should be elucidated in different cell/tissue models. This study demonstrates how the exosomal cargo of MSCs treated with Epo affects the miRNA content. However, it is another issue that needs to be elucidated about how this effect affects the content of exosomal cargo at the level of proteomics and metabolomes.

Considering the biological functions of MSCs such as immunomodulation and the function of supporting cells in the bone marrow niche, it is critical to reveal the contents of exosomes. Research in in vitro systems that mimic different physiological conditions and disease models will be important for MSC and exosome biology.

\section{ACKNOWLEDGEMENTS}

The authors would like to thank Assoc. Prof. Ömür Çelikbıçak for kindly providing Erythropoietin, Ekin Çelik and Can Koşukcu for technical assistance. This work is based on Stem Cell Sciences, Master of Science Thesis "Dilara Dalkıran, The Effect of Erythropoietin Treatment on Expression Profile of Mesenchymal Stem Cells, Hacettepe University Institute of Health Sciences, Stem Cell Sciences Program, Ankara, 2018." This work was partially supported by HÜ-BAP (TAY-2015-7335).

\section{CONFLICT Of INTEREST STATEMENT}

The authors declare that they have no conflict of interest.
[1] Kobolak J, Dinnyes A, Memic A, et al. Mesenchymal stem cells: Identification, phenotypic characterization, biological properties and potential for regenerative medicine through biomaterial micro-engineering of their niche. Methods 2016; 99: 62-8.

[2] Spees JL, Lee RH, Gregory CA. Mechanisms of mesenchymal stem/stromal cell function. Stem Cell Res Ther 2016; 7(1): 125.

[3] Vizoso FJ, Eiro N, Cid S, et al. Mesenchymal Stem Cell Secretome: Toward Cell-Free Therapeutic Strategies in Regenerative Medicine. Int J Mol Sci. 2017; 18(9).

[4] Thery C, Ostrowski M, Segura E. Membrane vesicles as conveyors of immune responses. Nat Rev Immunol 2009; 9(8): 581-93.

[5] Zhang H-G, Grizzle WE. Exosomes: A Novel Pathway of Local and Distant Intercellular Communication that Facilitates the Growth and Metastasis of Neoplastic Lesions. The American Journal of Pathology 2014; 184(1): 28-41.

[6] Ophelders DR, Wolfs TG, Jellema RK, et al. Mesenchymal Stromal Cell-Derived Extracellular Vesicles Protect the Fetal Brain After Hypoxia-Ischemia. Stem Cells Transl Med 2016; 5(6): 754-63.

[7] Collino F, Bruno S, Incarnato D, et al. AKI Recovery Induced by Mesenchymal Stromal Cell-Derived Extracellular Vesicles Carrying MicroRNAs. J Am Soc Nephrol 2015; 26(10): 2349-60

[8] Jarmalaviciute A, Pivoriunas A. Exosomes as a potential novel therapeutic tools against neurodegenerative diseases. Pharmacol Res 2016; 113(Pt B): 816-22.

[9] Tomasoni S, Longaretti L, Rota C, et al. Transfer of growth factor receptor mRNA via exosomes unravels the regenerative effect of mesenchymal stem cells. Stem Cells Dev 2013; 22(5): 772-80.

[10] Phinney DG, Pittenger MF. Concise Review: MSC-Derived Exosomes for Cell-Free Therapy. Stem Cells 2017; 35(4): 851-8.

[11] Miyake T, Kung CK, Goldwasser E. Purification of human erythropoietin. J Biol Chem 1977; 252(15): 5558-64.

[12] Onal EM, Sag AA, Sal O, et al. Erythropoietin mediates brain-vascular-kidney crosstalk and may be a treatment target for pulmonary and resistant essential hypertension. Clin Exp Hypertens 2017; 39(3): 197-209.

[13] Powell JS, Berkner KL, Lebo RV, et al. Human erythropoietin gene: high level expression in stably transfected mammalian cells and chromosome localization. Proc Natl Acad Sci USA 1986; 83(17): 6465-9.

[14] Noguchi CT, Wang L, Rogers HM, el at. Survival and proliferative roles of erythropoietin beyond the erythroid lineage. Expert Rev Mol Med 2008; 10: e36.

[15] Maiese K, Li F, Chong ZZ. New avenues of exploration for erythropoietin. JAMA 2005; 293(1): 90-5.

[16] Li J, Guo W, Xiong M, et al. Erythropoietin facilitates the recruitment of bone marrow mesenchymal stem cells to sites of spinal cord injury. Exp Ther Med 2017; 13(5): 1806-12.

[17] Wang Y, Lu X, He J, et al. Influence of erythropoietin on 
microvesicles derived from mesenchymal stem cells protecting renal function of chronic kidney disease. Stem Cell Res Ther 2015; 6: 100.

[18] Tari K, Atashi A, Kaviani S, et al. Erythropoietin induces production of hepatocyte growth factor from bone marrow mesenchymal stem cells in vitro. Biologicals 2017; 45: 15-9.

[19] Ercan E, Bagla AG, Aksoy A, et al. In vitro protection of adipose tissue-derived mesenchymal stem cells by erythropoietin. Acta Histochem 2014; 116(1): 117-25.

[20] Koh SH, Noh MY, Cho GW, et al. Erythropoietin increases the motility of human bone marrow-multipotent stromal cells (hBM-MSCs) and enhances the production of neurotrophic factors from hBM-MSCs. Stem Cells Dev 2009; 18(3): 411-21.

[21] Liao Y-C, Wang Y-S, Guo Y-C, et al. Let-7g Improves Multiple Endothelial Functions Through Targeting Transforming Growth Factor-Beta and SIRT-1 Signaling. Journal of the American College of Cardiology 2014; 63(16): 1685-94.

[22] Zhang Y, Fan M, Zhang X, et al. Cellular microRNAs up-regulate transcription via interaction with promoter TATAbox motifs. RNA 2014; 20(12): 1878-89.

[23] Zhang J, Ma J, Long K, et al. Overexpression of Exosomal Cardioprotective miRNAs Mitigates Hypoxia-Induced H9c2 Cells Apoptosis. Int J Mol Sci 2017; 18(4).

[24] Beltrami C, Besnier M, Shantikumar S, et al. Human Pericardial Fluid Contains Exosomes Enriched with Cardiovascular-Expressed MicroRNAs and Promotes Therapeutic Angiogenesis. Molecular therapy: the journal of the American Society of Gene Therapy 2017; 25(3): 679-93.

[25] Yang H, Fang F, Chang R, et al. MicroRNA-140-5p suppresses tumor growth and metastasis by targeting transforming growth factor beta receptor 1 and fibroblast growth factor 9 in hepatocellular carcinoma. Hepatology 2013; 58(1): 205-17.

[26] Si X, Zhang X, Hao X, et al. Upregulation of miR-99a is associated with poor prognosis of acute myeloid leukemia and promotes myeloid leukemia cell expansion. Oncotarget 2016; 7(47): 78095-109.

[27] Chen C-F, He X, Arslan AD, et al. Novel regulation of NFYB by miR-485-3p affects expression of DNA topoisomerase lla and drug responsiveness. Molecular Pharmacology 2011; 79(4): 735-41.

[28] Li Y, Chen D, Li Y, et al. Oncogenic CAMP responsive element binding protein 1 is overexpressed upon loss of tumor suppressive miR-10b-5p and miR-363-3p in renal cancer. Oncol Rep 2016; 35(4): 1967- 78.
[29] Liu Z-R, Song Y, Wan L-H, et al. Over-expression of miR451a can enhance the sensitivity of breast cancer cells to tamoxifen by regulating 14-3-3 , estrogen receptor $a$, and autophagy. Life Sciences 2016; 149: 104-13.

[30] Rasheed Z, Al-Shobaili HA, Rasheed N, et al. MicroRNA$26 a-5 p$ regulates the expression of inducible nitric oxide synthase via activation of NF-KB pathway in human osteoarthritis chondrocytes. Archives of Biochemistry and Biophysics 2016; 594: 61-7.

[31] Wang X, Liu S, Cao L, et al. miR-29a-3p suppresses cell proliferation and migration by downregulating IGF1R in hepatocellular carcinoma. Oncotarget 2017; 8(49): 86592-603.

[32] Wang T, Ren Y, Liu R, et al. miR-195-5p Suppresses the Proliferation, Migration, and Invasion of Oral Squamous Cell Carcinoma by Targeting TRIM14. Biomed Res Int 2017; 2017: 7378148.

[33] Dong W, Yao C, Teng X, et al. MiR-140-3p suppressed cell growth and invasion by downregulating the expression of ATP8A1 in non- small cell lung cancer. Tumor Biology 2016; 37(3): 2973-85.

[34] Yang XW, Zhang LJ, Huang XH, et al. miR-145 suppresses cell invasion in hepatocellular carcinoma cells: miR-145 targets ADAM17. Hepatol Res 2014; 44(5): 551-9.

[35] Hsu KW, Fang WL, Huang KH, et al. Notch1 pathway-mediated microRNA-151-5p promotes gastric cancer progression. Oncotarget 2016; 7(25): 38036-51.

[36] Geng L, Sun B, Gao B, et al. MicroRNA-103 promotes colorectal cancer by targeting tumor suppressor DICER and PTEN. International journal of molecular sciences 2014; 15(5): 8458-72.

[37] Yang J, Zhang Z, Chen C, et al. MicroRNA-19a-3p inhibits breast cancer progression and metastasis by inducing macrophage polarization through downregulated expression of Fra-1 proto-oncogene. Oncogene 2013; 33: 3014.

[38] Busch S, Auth E, Scholl F, et al. 5- lipoxygenase is a direct target of miR-19a-3p and miR-125b-5p. J Immunol 2015; 194(4): 1646-53.

[39] Wang J, He Q, Han C, et al. p53-facilitated miR-199a-3p regulates somatic cell reprogramming. Stem Cells 2012; 30(7): 1405-13.

[40] Liu J, Wang Y, Cui J, et al. miR199a-3p regulates P53 by targeting CABLES1 in mouse cardiac c-kit(+) cells to promote proliferation and inhibit apoptosis through a negative feedback loop. Stem Cell Res Ther 2017; 8(1): 127. 